\title{
Pre-eclampsia: evidence of altered ventricular repolarization by standard ECG parameters and QT dispersion
}

\author{
Ricciarda Raffaelli ${ }^{1}$, Maria Antonia Prioli ${ }^{2}$, Francesca Parissone ${ }^{1}$, Daniele Prati ${ }^{2}$, Michela Carli ${ }^{1}$, \\ Corinna Bergamini ${ }^{2}$, Giuseppe Cacici $^{2}$, Debora Balestreri ${ }^{1}$, Corrado Vassanelli ${ }^{2}$ and Massimo Franchi $^{1}$
}

Pre-eclampsia complicates approximately $6-8 \%$ of all pregnancies. Epidemiologic studies have demonstrated a relationship between pre-eclampsia and cardiac morbidity and mortality later in life, but the effect of pre-eclampsia on electrical cardiac activity during the acute phase has not yet been understood. The aim of this study was to investigate ECG alterations during pre-eclampsia. Prepartum ECGs of 76 consecutive pre-eclamptic women were compared with those of 76 healthy pregnant women. All of the routine ECG parameters were considered, and ventricular repolarization was assessed by QT interval and QT dispersion (QTd). Pregnancies complicated by pre-eclampsia showed a significant alteration of ventricular repolarization compared with the control group. Among ECG parameters, QT and QTc intervals and QTd were more prolonged in pre-eclamptic women. Multivariate analysis also showed that pre-eclampsia was the only independent determinant of QTd. In conclusion, pre-eclampsia has a significant effect on ventricular repolarization. This alteration could, in part, explain the increased cardiovascular risk of women with a history of pre-eclampsia. Further studies are necessary to confirm the relationship between ventricular repolarization abnormalities and increased cardiovascular risk later in life.

Hypertension Research (2014) 37, 984-988; doi:10.1038/hr.2014.102; published online 26 June 2014

Keywords: electrocardiogram; pre-eclampsia; QT dispersion; QTc interval; ventricular repolarization

\section{INTRODUCTION}

Hypertensive disorders during pregnancy affect $6-8 \%$ of all pregnancies, and pre-eclampsia is one of the leading causes of maternal and fetal morbidity and mortality. ${ }^{1}$ In particular, preeclampsia is associated with maternal life-threatening conditions, such as eclampsia, HELLP (hemolysis, elevated liver enzymes, low platelets) syndrome, disseminated intravascular coagulation, pulmonary edema, abruption placentae and hemorrhage, as well as neonatal complications such as preterm delivery, fetal growth restriction, hypoxic-neurologic injury and perinatal death. ${ }^{2}$ In addition, many studies have demonstrated that women with a history of pre-eclampsia have an increased risk of subsequent cardiac, cerebrovascular and peripheral arterial diseases. These women are more likely than the rest of the population to develop cardiovascular diseases (myocardial infarction, stroke, hypertension) with an earlier onset. ${ }^{3-5}$ An early onset of pre-eclampsia, greater severity of disease or recurrence in a second pregnancy seem to further exacerbate this risk. ${ }^{3,6,7}$ The mechanisms underlying this increased cardiovascular risk have not yet been understood. According to some authors, pre-eclampsia and cardiovascular disease simply shares the same individual predisposition and risk factors. ${ }^{8,9}$ Others believe that pre-eclampsia itself causes or at least aggravates cardiovascular diseases. ${ }^{3}$ Nonetheless, the acute effect of preeclampsia on the heart is not well known.

Previous studies have focused on echocardiographic changes during pregnancy-induced hypertension and have shown a higher prevalence of pathological findings such as increased size of the atrium and left ventricle, a thicker interventricular septum and pericardial effusion. ${ }^{10-12}$ Pregnancy induces hemodynamic, metabolic and endocrine changes that impact cardiac function causing ECG alterations such as prolonged QTc and leftward deviation of the QRS and T axes. ${ }^{13,14}$ The role of a standard ECG in detecting asymptomatic signs of cardiac damage during pregnancy complicated by pre-eclampsia is controversial. A previous study has reported evidence of an alteration in ECG parameters relative to ventricular repolaritazion (QT and QTc intervals) in women with abnormal uterine perfusion and subsequent development of pre-eclampsia. ${ }^{15}$ QT-interval parameters, in particular, heart rate-corrected QT interval duration, are presumed markers of increased cardiovascular risk and provide important prognostic information in clinical practice. ${ }^{16}$

${ }^{1}$ Department of Life and Reproduction Sciences, Obstetrics and Gynecology Section, University of Verona, Verona, Italy and ${ }^{2}$ Department of Medicine, Cardiology Section, University of Verona, Verona, Italy

Correspondence: Dr R Raffaelli, Department of Life and Reproduction Sciences, Obstetrics and Gynecology Section, University of Verona, Policlinico GB Rossi, Piazzale LA Scuro 10, Verona 37134, Italy.

E-mail: ricciarda.raffaelli@univr.it

Received 10 June 2013; revised 14 April 2014; accepted 25 April 2014; published online 26 June 2014 
QT dispersion (QTd) is defined as the difference between the maximum and the minimum QT interval measured in each ECG lead. Different ECG leads magnify the ECG signal of different myocardial regions, and as a result, QTd is almost a direct measure of the heterogeneity of myocardial repolarization. ${ }^{17,18}$

Increased QTd is believed to be associated with a major risk of arrhythmias, and many studies have tested its usefulness as a prognostic factor in several diseases. The aim of this study is to establish if standard ECG parameters and QTd could give additional information about the acute cardiac effects of pre-eclampsia, even in the absence of symptoms.

\section{METHODS}

\section{Subjects}

Women affected by pre-eclampsia $(n=76)$ who delivered at GB Rossi Policlinic of Verona, Italy, from 1 January 2010 to 31 December 2011 were included in the study group. Inclusion criteria for the study group were the following: a diagnosis of pre-eclampsia, HELLP (hemolysis, elevated liver enzymes, low platelets) syndrome, eclampsia and a singleton pregnancy. Seventy-six consecutive pregnant women without cardiovascular disease or gestational hypertension who delivered at our hospital starting from 1 January 2010 were considered for the control group; twin pregnancies were excluded. Maternal age was comparable between both groups (pre-eclampsia group: $32.2 \pm 6.3$ years; control group: $32.2 \pm 5.7$ years). In a second phase of the study, pre-eclamptic women were further divided into 'early-onset preeclampsia' (46 subjects) and 'late-onset pre-eclampsia' (30 subjects) subgroups as defined previously. ${ }^{19-21}$ These two groups were comparable with regard to the same ECG parameters. The diagnostic criteria for pre-eclampsia, eclampsia and HELLP syndrome were those outlined by the National High Blood Pressure Education Program (NHBPEP) working group. ${ }^{22}$

\section{ECG}

A standard 12-lead ECG was performed on all patients before delivery. All ECGs were recorded using the P8000 Power Esaote ECG recorder and analyzed by the Cardiology Org@nizer-Esaote analysis software (Esaote SpA, Genoa,
Italy). The software analyzed the following standard ECG parameters: heart rate (b.p.m.), RR interval (ms), QT interval (ms), corrected QT interval (ms), QRS complex duration (ms) and P-wave duration (ms). In addition, QTd was determined on each ECGs. QTd was obtained by calculating the difference between the maximum and the minimum QT interval measured in each ECG lead from the onset of QRS complex to the end of T wave. ${ }^{23}$ The QT interval was manually measured by a single investigator who was unaware of the clinical outcomes, using an electronic caliper on ECG tracing displayed on a digital screen and magnified (Figure 1). Moreover, each ECG was analyzed by a cardiologist for common pathologic findings such as the following: ST changes (ST segment depression or elevation $\geqslant 1 \mathrm{~mm}$, flattening of the $\mathrm{T}$ wave, biphasic $\mathrm{T}$ waves or T-wave inversion), arrhythmias and other HR alterations, left atrial enlargement and left ventricular hypertrophy, cardiac axis deviation. Each of the previously described ECG parameters was compared between the preeclamptic women and the control group, as well as between 'early-onset' and 'late-onset' pre-eclampsia subgroups.

\section{Other clinical and instrumental data}

Clinical and laboratory parameters were also collected from medical records. Blood pressure (BP) was measured using a manual standard sphygmomanometer. The systolic BP (SBP) and diastolic BP (DBP) recorded while obtaining the ECG were taken into consideration. Antihypertensive therapy and magnesium sulfate $\left(\mathrm{MgSO}_{4}\right)$ treatment to prevent seizures in severe preeclampsia were regarded as possible determinants of the ECG parameters. Finally, other clinical information such as body mass index, gestational diabetes, current smoking status and $\mathrm{K}^{+}$serum concentration at the time the ECG was obtained and analyzed.

\section{Data analysis}

Statistical analysis was performed using SPSS (Statistical Package for the Social Sciences) version 11.0 software (SPSS, Chicago, IL, USA). Data are reported as the mean \pm s.d. for quantitative variables or percentage for qualitative ones; only QTd is reported as the median (25th-75th percentile). Non-normal and normally distributed data were analyzed using the Mann-Whitney $U$-test and unpaired Student's $t$-test, respectively. Binomial data were analyzed using the $\chi^{2}$ test. In addition, a multivariate analysis was performed using a generalized

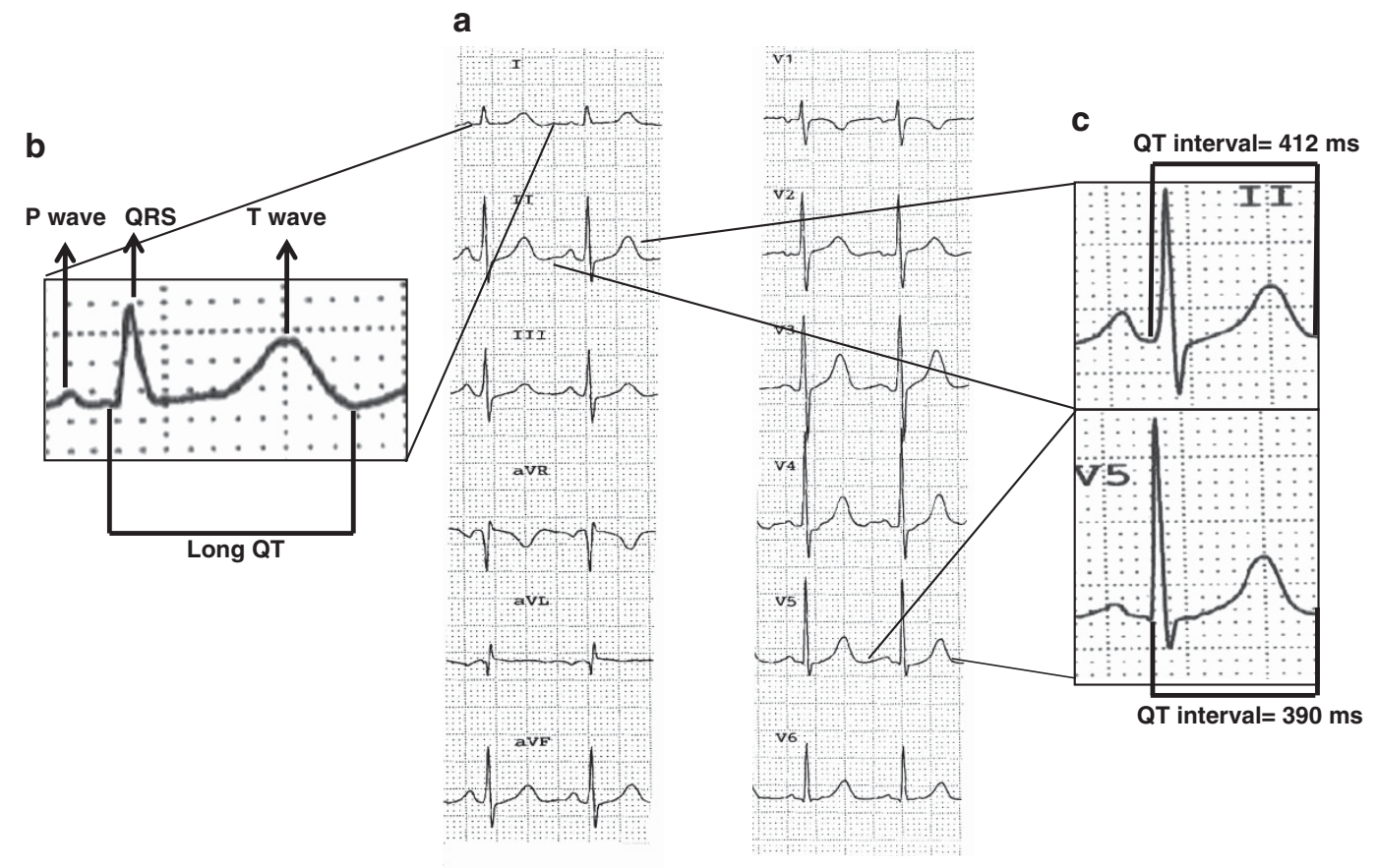

Figure 1 (a) A standard 12-lead ECG was performed on all patients before delivery. (b) Example of a long QT interval. (c) Example of QT dispersion calculated as the difference between maximum and minimum QT interval measured on each ECG leads. 
Table 1 Subject characteristics and ECG parameters of women with pre-eclampsia and women in the control group

\begin{tabular}{|c|c|c|c|}
\hline Subject characteristics & Pre-eclampsia & Control group & P-value \\
\hline Maternal age (years) & $32.2 \pm 6.3$ & $32.2 \pm 5.7$ & 0.890 \\
\hline $\mathrm{BMI}\left(\mathrm{kg} \mathrm{m}^{-2}\right)$ & $24.9 \pm 5.2$ & $22.2 \pm 3.1$ & 0.002 \\
\hline $\mathrm{SBP}(\mathrm{mm} \mathrm{Hg})$ & $156.8 \pm 17.8$ & $117.2 \pm 11.7$ & $<0.001$ \\
\hline $\mathrm{DBP}(\mathrm{mm} \mathrm{Hg})$ & $101.2 \pm 8.8$ & $73.1 \pm 8.3$ & $<0.001$ \\
\hline $\begin{array}{l}\text { Gestational age at delivery } \\
\text { (weeks) }\end{array}$ & $34.4 \pm 3.4$ & $38.5 \pm 2.1$ & $<0.001$ \\
\hline Potassium (mmoll-1) & $4.1 \pm 0.4$ & $3.8 \pm 0.3$ & $<0.001$ \\
\hline Current smokers (\%) & 9.2 & 27.6 & 0.006 \\
\hline Gestational diabetes (\%) & 11.8 & 11.8 & 1.000 \\
\hline Labetalol os (\%) & 10.5 & - & - \\
\hline Labetalol i.v. (\%) & 82.9 & - & - \\
\hline No antihypertensive therapy & 6.6 & & \\
\hline $\mathrm{MgSO}_{4}(\%)$ & 46.1 & - & - \\
\hline \multicolumn{4}{|l|}{$E C G$} \\
\hline $\mathrm{HR}(\mathrm{ms})$ & $77.4 \pm 14.3$ & $81.6 \pm 11.0$ & 0.005 \\
\hline RR interval (ms) & $806.8 \pm 158.5$ & $743.2 \pm 99.2$ & 0.002 \\
\hline QRS complex (ms) & $83.2 \pm 8.1$ & $83.6 \pm 8.3$ & 0.917 \\
\hline P-wave duration (ms) & $100.3 \pm 7.6$ & $98.8 \pm 9.2$ & 0.042 \\
\hline QT interval (ms) & $395.6 \pm 40.5$ & $361.4 \pm 41.3$ & $<0.001$ \\
\hline \multirow[t]{2}{*}{ QTC interval (ms) } & $442.7 \pm 26.7$ & $423.68 \pm 20.7$ & $<0.001$ \\
\hline & $\begin{array}{c}\text { Median } \\
\text { (25th-75th } \\
\text { percentile) }\end{array}$ & $\begin{array}{c}\text { Median } \\
\text { (25th-75th } \\
\text { percentile) }\end{array}$ & P-value \\
\hline
\end{tabular}

QTd (ms)

$24.0(22.0-43.0) \quad 22.0(20.0-22.0) \quad<0.001$

Abbreviations: $\mathrm{BMI}$, body mass index; $\mathrm{DBP}$, diastolic blood pressure; $\mathrm{HR}$, heart rate; $\mathrm{MgSO}_{4}$ magnesium sulfate; QTc, corrected QT interval; QTd, QT dispersion; SBP, systolic blood pressure.

All data are reported as mean \pm s.d. except QTd, which is reported as median (25th-75th percentile).

linear model to exclude other determinants of QTc interval and QTd. A $P$-value $<0.05$ was considered statistically significant.

\section{RESULTS}

Comparison between pre-eclamptic patients and the control group Subject characteristics and clinical/laboratory data of the two groups are reported in Table 1.

There were significant differences in the following data: SBP, DBP, $\mathrm{K}^{+}$serum concentration and body mass index.

The routine evaluation of the ECGs in both groups was mostly nonpathologic, and there were no significant differences between any of the pathologic findings considered. ECG routine parameters provided by the ECG software are reported in Table 1. Pre-eclamptic women showed a lower HR than the control group $(P=0.005)$, and consequently, a longer RR interval $(P=0.002)$. Labetalol, an $\alpha / \beta$ blocker, was used to treat $93.4 \%$ of patients. P-wave duration, QT interval and QTc interval were significantly longer in the pre-eclamptic women than in the control group $(P<0.05)$. QTd was significantly higher in pre-eclamptic women than in the control group $(P<0.001)$ (Figure 2$)$

\section{Comparison between 'early-onset' and 'late-onset' pre-eclampsia subgroups}

There were no significant differences between the two subgroups for any ECG and cardiovascular parameters considered (Table 2).

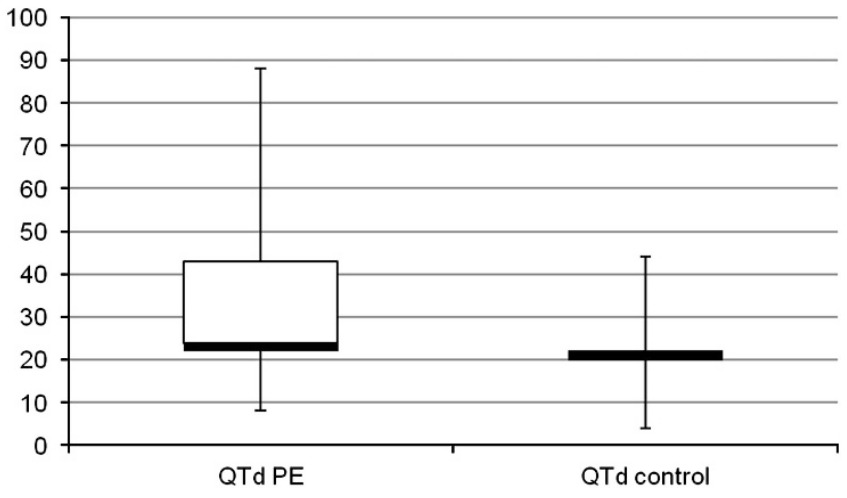

Figure 2 Comparison between QT dispersion (QTd) in pre-eclamptic women and pregnant women without pre-eclampsia.

Table 2 Cardiovascular and ECG parameters of women with 'early-onset' and 'late-onset' pre-eclamspia

\begin{tabular}{lccc}
\hline & Early-onset & Late-onset & P-value \\
\hline SBP & $159.3 \pm 14.3$ & $154.1 \pm 21.2$ & 0.824 \\
DBP & $100.3 \pm 7.1$ & $102.3 \pm 10.5$ & 0.185 \\
HR (b.p.m.) & $79.3 \pm 14.8$ & $75.4 \pm 14.0$ & 0.414 \\
RR interval (ms) & $790.5 \pm 157.6$ & $825.0 \pm 162.2$ & 0.918 \\
QRS complex (ms) & $84.0 \pm 8.6$ & $82.2 \pm 7.7$ & 0.514 \\
P-wave duration (ms) & $100.1 \pm 8.7$ & $100.6 \pm 6.4$ & 0.526 \\
QT interval (ms) & $395.6 \pm 41.9$ & $395.6 \pm 40.1$ & 0.893 \\
QTc interval (ms) & $447.3 \pm 24.4$ & $437.6 \pm 28.9$ & 0.524 \\
QTd (ms) & 32.4 & 35.3 & 0.367
\end{tabular}

Abbreviations: DBP, diastolic blood pressure; HR, heart rate; QTc, corrected QT interval; QTd, QT dispersion; SBP, systolic blood pressure;

\section{Multivariate analysis}

To understand the determinants of QTd and QTc, a multiple regression analysis was performed.

The following data were considered as independent variables: diagnosis of pre-eclampsia, SBP and DBP measured at the time of ECG was obtained, $\mathrm{K}^{+}$serum concentration, body mass index, current smoking status, gestational diabetes and $\mathrm{MgSO}_{4}$ treatment to prevent seizures.

A diagnosis of pre-eclampsia was the only significant determinant of QTd $(P=0.017)$ and was associated with an increased QTd (unstandardized coefficient $B=9.80 ; 95 \%$ confidence interval = 1.21-18.40). Statistically significant determinants of QTc were SBP $(B=0.28 ; 95 \%$ confidence interval $=0.04-0.51 ; P=0.020)$ and $\mathrm{MgSO}_{4}$ treatment $(P<0.001)$, with the latter seeming to induce an increased QTc $(B=26.745 ; 95 \%$ confidence interval $=16.84-36.64)$. As shown in Figure 2, the QTc interval was longer in patients who had been treated with $\mathrm{MgSO}_{4}$ (451.3 vs $424.4 \mathrm{~ms} ; P<0.001$ ).

\section{DISCUSSION}

In this study, women with pre-eclampsia demonstrated a longer P-wave, lower HR, longer QT and QTc intervals, and higher QTd compared with the control group. However, the ECG of both preeclamptic patients and the control group were mostly nonpathologic, a finding consistent with a previous study that investigated the prevalence of ECG abnormalities after pregnancies complicated by pre-eclampsia. $^{24}$

The P-wave duration, which reflects the size of the atria, was found to be longer in women with pre-eclampsia. ${ }^{25}$ Left atrial enlargement is 
very common in hypertensive disorders and reflects the presence of left ventricular diastolic dysfunction due to chronic high BP levels. ${ }^{25-27} \mathrm{~A}$ recent study has proposed the identification of an abnormal morphology of the $\mathrm{P}$ wave in lead $\mathrm{V} 1$ during pregnancy as a possible predictor of the development of a hypertensive disorder. ${ }^{28}$ In our study, the P-wave duration was slightly longer in patients with pre-eclampsia than in the control group, but with a weak statistical significance. This finding may, in part, be due to the effect of antihypertensive therapy on reducing left ventricular afterload with consequent lower left atrial enlargement. Women with pre-eclampsia also showed a tendency toward lower heart rates compared with the controls, a finding that may be explained by the effect of labetalol, an $\alpha / \beta$ blocker.

\section{Alteration and heterogeneity of the ventricular repolarization process during pre-eclampsia}

In this study QT, QTc and QTd significantly differed between the two groups. It is possible that pre-eclampsia has a significant effect on ventricular repolarization. A recent study has shown a trend towards shorter QTc intervals in the second half of gestation, before the onset of clinical symptoms in pregnancies with abnormal uterine perfusion that developed pre-eclampsia or delivered a small-for-gestational age infant. ${ }^{15}$ In contrast, in a previous prospective study, women who developed eclampsia showed a significantly longer QTc interval. ${ }^{29}$ In this study, both QT and QTc intervals of pre-eclamptic women were more prolonged than those of the control group. It is important to emphasize that only ECG parameters recorded at the end of pregnancy during the acute phase of the disease were considered. The main consequence of a long QTc is the risk of arrhythmias, a risk that becomes real when QTc is $>500 \mathrm{~ms}^{30}$ In this study, only one pre-eclamptic woman had a QTc $>500 \mathrm{~ms}$, and none had clinical arrhythmias.

The multivariate analysis also showed that the determinants of prolonged QTc were $\mathrm{MgSO}_{4}$ treatment and the SBP. According to the literature, the hypermagnesemia $\left(\mathrm{Mg}^{2+}>0.95 \mathrm{mmoll}^{-1}\right)$ that may result from treatment with high doses of $\mathrm{MgSO}_{4}$ can cause a lengthening of the QT and QTc intervals. ${ }^{31,32}$

To our knowledge, this is the first study investigating QTd during pre-eclamspia. QTd is considered a marker of heterogeneity of ventricular repolarization. ${ }^{23}$ Many studies have tested its usefulness as a predictor of cardiovascular mortality in several clinical conditions. ${ }^{33-39}$ In this study, pre-eclamptic women showed a higher dispersion of QT interval than the control group, confirming the alteration of ventricular repolarization in these women. Moreover, as demonstrated by multivariate analysis, the only significant determinant of QTd was the presence of pre-eclampsia itself.

The heterogeneity of the ventricular repolarization phase underlies electrophysiological alterations that occur during pre-eclampsia, even in the absence of symptoms. Previous studies have focused on the effect of pre-eclampsia on heart rate variability and have shown a state of sympathetic hyperactivity, especially before the onset of symptoms. ${ }^{40,41}$ A recent study has suggested that placental factors, such as soluble fms-like tyrosine kinase-1, soluble endoglin and placental growth factor, which are supposed to be involved in the pathogenesis of pre-eclampsia, could cause vasoconstriction by acting on the peripheral and central nervous systems. ${ }^{42-44}$ The electrophysiologic cardiac alterations may be caused by the increased hemodynamic stress on the cardiovascular system owing to the high SBP. However, mediators circulating at high levels during pre-eclampsia may also have a specific role. ${ }^{15,33}$
Thus far, the relationship between pre-eclampsia and a higher cardiovascular risk later in life is well demonstrated. ${ }^{3-5}$ Previous studies have shown that prolonged ventricular repolarization is a risk factor for ischemic heart disease and cardiovascular mortality in subjects with uncomplicated hypertension. ${ }^{45,39}$ Alteration of ECG parameters such as QTc and QTd could be the earliest sign of asymptomatic ventricular electrical instability that could, in part, explain the increased future risk of cardiovascular disease in pre-eclamptic women.

Early-onset pre-eclampsia is usually characterized by fetal growth restriction and more severe maternal disease, whereas late-onset preeclampsia is more common and is usually associated with normal birth weight. Researchers have suggested that early- and late-onset pre-eclampsia may be distinct entities with different etiologies, ${ }^{19-21}$ and there seem to be two different hemodynamic states underlying these two forms. ${ }^{46}$ Moreover, women with a history of severe preeclampsia, particularly those who experienced preterm delivery, poor fetal growth or fetal death, seem to have an increased cardiovascular risk. ${ }^{3}$ A recent study has shown that preterm pre-eclampsia (delivery before 37 weeks gestation) is strongly associated with the persistence of left ventricular dysfunction/hypertrophy at the 1 year postpartum follow-up. ${ }^{6}$ However, in our experience, the time of disease onset does not seem to worsen the electrocardiographic alterations.

\section{Limitations of the study}

In most cases, QTd is still measured manually, which explains the high inter- and intraobserver variability. ${ }^{23}$ In this study, we tried to limit this variability by using a digitizing board and an electronic caliper, as well as by engaging a single investigator, who was unaware of the clinical outcomes. Several studies have tried to validate automatic algorithms to measure QTd, and recent software includes the measurement of QTd. This implementation might allow for easier use of this parameter in clinical practice. ${ }^{47}$

\section{Conclusion and future implications}

Pre-eclampsia induces an alteration of ventricular repolarization as evidenced by prolongation of ECG parameters, such as QT, QTc and QTd. Pre-eclampsia seems to be the sole determinant of the increased QTd. Although these changes are mostly asymptomatic, the simple, noninvasive assessment of ventricular repolarization on a standard 12-lead ECG could be easily included in the clinical evaluation of pre-eclamptic women. Further studies are needed, however, to demonstrate the relationship between abnormalities of ventricular repolarization and increased cardiovascular risk in these patients, so that the appropriate cutoff for risk stratification can be defined.

\section{CONFLICT OF INTEREST}

The authors declare no conflict of interest.

\section{ACKNOWLEDGEMENTS}

We are grateful to Prof Poli for advice on the statistical analyses.

1 ACOG. (2002) American College of Obstetricians and Gynecologists practice bulletin. Diagnosis and management of preeclampsia and eclampsia. Int J Gynaecol Obstet 2002; 77: 67-75.

2 Sibai BM. Pre-eclampsia. Lancet 2005; 365: 785-799.

3 McDonald SD, Malinowski A, Zhou Q, Yusuf S, Devereaux PJ. Cardiovascular sequelae of preeclampsia/eclampsia: a systematic review and meta-analyses. Am Heart J 2008; 156: 918-930.

4 Bellamy L, Casas JP, Hingorani AD, Williams DJ. Pre-eclampsia and risk of cardiovascular disease and cancer in later life: systematic review and meta-analysis. BMJ 2007; 335: 974. 
5 Evans CS, Gooch L, Flotta D, Lykins D, Powers RW, Landsittel D, Roberts JM, Shroff SG. Cardiovascular system during the postpartum state in women with a history of preeclampsia. Hypertension 2011; 58: 57-62.

6 Melchiorre K, Sutherland GR, Baltabaeva A, Liberati M, Thilaganathan B. Maternal cardiac dysfunction and remodeling in women with preeclampsia at term. Hypertension 2011; 57: 85-93.

7 Sep SJ, Schreurs MP, Bekkers SC, Kruse AJ, Smits LJ, Peeters LL. Early-pregnancy changes in cardiac diastolic function in women with recurrent pre-eclampsia and in previously pre-eclamptic women without recurrent disease. BJOG 2011; 118: 1112-1119.

8 Magnussen EB, Vatten LJ, Lund-Nilsen TI, Salvesen KA, Davey Smith G, Romundstad PR. Prepregnancy cardiovascular risk factors as predictors of preeclampsia: population based cohort study. BMJ 2007; 335: 978.

9 Rodie VA, Freeman DJ, Sattar N, Greer IA. Pre-eclampsia and cardiovascular disease: metabolic syndrome of pregnancy. Atherosclerosis 2004; 175 189-202.

10 Yuan L, Duan Y, Cao T. Echocardiographic study of cardiac morphological and functional changes before and after parturition in pregnancy-induced hypertension. Echocardiography 2006; 23: 177-182.

11 Vasapollo B, Novelli GP, Valensise H. Total vascular resistance and left ventricular morphology as screening tools for complications in pregnancy. Hypertension 2008; 51: 1020-1026.

12 Novelli GP, Valensise H, Vasapollo B, Larciprete G, Altomare F, Di Pierro G, Casalino B, Galante A, Arduini D. Left ventricular concentric geometry as a risk factor in gestational hypertension. Hypertension 2003; 41: 469-475.

13 Carruth JE, Mivis SB, Brogan DR, Wenger NK. The electrocardiogram in normal pregnancy. Am Heart J 1981; 102: 1075-1078.

14 Lechmanová M, Parízek A, Halaska M, Slavícek J, Kittnar O. Changes of the electrical heart field and hemodynamic parameters in the 34th to 40th weeks of pregnancy and after delivery. Arch Gynecol Obstet 2002; 266: 145-151.

15 Baumert M, Seeck A, Faber R, Nalivaiko E, Voss A. Longitudinal changes in QT interval variability and rate adaptation in pregnancies with normal and abnormal uterine perfusion. Hypertens Res 2010; 33: 555-560.

16 Dekker JM, Schouten EG, Klootwijk P, Pool J, Krombout D. Association between QT interval and coronary heart disease in middle-aged and elderly men: the Zutphen study. Circulation 1994; 90: 779-785.

17 Wilson FN, Macleod AG, Barker PS, Johnston FD. Determination of the signficance of the areas of the ventricular deflections of the electrocatdiogram. Am Heart J 1934; 10 46.

18 Day CP, Mc Comp LM, Compbell R. QT dispersion: an indication of arrhythmia risk in patients with long QT intevals. Br Heart J 1990; 63: 342-344.

19 Eastabrook G, Brown M, Sargent I. The origins and end-organ consequence of pre-eclampsia. Best Pract Res Clin Obstet Gynaecol 2011; 25: 435-447.

20 von Dadelszen P, Magee LA, Roberts JM. Subclassification of preeclampsia. Hypertens Pregnancy 2003; 22: 143-148.

21 Huppertz B. Placental origins of preeclampsia: challenging the current hypothesis. Hypertension 2008; 51: 970-975.

22 Roccella EJ. Report of the National High Blood Pressure Education Program Working Group on High Blood Pressure in Pregnancy (NHBPEP). Am J Obstet Gynecol 2000; 183: S1-S22

23 Malik M, Batchvarov VN. Measurement, interpretation and clinical potential of QT dispersion. J Am Coll Cardiol 2000; 36: 1749-1766.

24 Hoogsteder PH, Krüse AJ, Sep SJ, Dassen WR, Gorgels AP, Peeters LL. Electrocardiographic findings in women with a recent history of pre-eclampsia. Acta Obstet Gynecol Scand 2012; 91: 372-378.

25 Miller JT, O'Rourke RA, Crawford MH. Left atrial enlargement: an early sign of hypertensive heart disease. Am Heart J 1988; 116: 1048.51.

26 Rossi A, Cicoira M, Zanolla L, Sandrini R, Golia G, Zardini P, Enriquez-Sarano M. Determinants and prognostic value of left atrial volume in patients with dilated cardiomyopathy. J Am Coll Cardiol 2002; 40: 1425.
27 Vaziri SM, Larson MG, Lauer MS, Benjamin EJ, Levy D. Influence of blood pressure on left atrial size. The Framingham Heart Study. Hypertension 1995; 26: 1155-1160.

28 Angeli E, Verdecchia P, Narducci P, Angeli F. Additive value of standard ECG for the risk prediction of hypertensive disorders during pregnancy. Hypertens Res 2011; 34: 707-713.

29 Isezuo SA, Ekele BA. Eclampsia and abnormal QTc. West Afr J Med 2004; 23. 123-127.

30 Bednar MM, Harrigan EP, Anziano RJ, Camm AJ, Ruskin JN. The QT interval. Prog Cardiovasc Dis 2001; 43: 1-45.

31 AA.VV. Resuscitation Council UK. ALS Manual, 6th edn. RESUSCITATION COUNCIL (UK) TRADING LTD: London.

32 Nakaigawa Y, Akazawa S, Shimizu R, Ishii R, Ikeno S, Inoue S, Yamato R. Effects of magnesium sulphate on the cardiovascular system, coronary circulation and myocardial metabolism in anaesthetized dogs. Br J Anaesth 1997; 793: 363-368.

33 Okin PM, Devereux RB, Howard BV, Fabsitz RR, Lee ET, Welty TK. Assessment of QT interval and QT dispersion for prediction of all-cause and cardiovascular mortality in American Indians: The Strong Heart Study. Circulation 2000; 101: 1.

34 Di lorio B, Bellasi A. Impact of vascular calcification on QT interval and QT dispersion in CKD and dialysis patients. Am J Nephrol 2012; 35: 287

35 Giunti S, Gruden G, Fornengo P, Barutta F, Amione C, Ghezzo G, Cavallo-Perin P, Bruno G. Increased QT interval dispersion predicts 15-year cardiovascular mortality in type 2 diabetic subjects: the population-based Casale Monferrato Study. Diabetes Care 2012; 35: 581-583.

36 Pan KL, Hsu JT, Chang ST, Chung CM, Chen MC. Prognostic value of QT dispersion change following primary percutaneous coronary intervention in acute ST elevation myocardial infarction. Int Heart J 2011; 52: 207-211.

37 Soydinc S, Davutoglu V, Akcay M. Uncomplicated metabolic syndrome is associated with prolonged electrocardiographic QTc interval and QTc dispersion. Ann Noninvasive Electrocardiol 2006; 11: 313-317.

38 Voigt L, Haq SA, Mitre CA, Lombardo G, Kassotis J. Effect of obstructive sleep apnea on QT dispersion: a potential mechanism of sudden cardiac death. Cardiology 2011; 118: 68-73.

39 Schillaci G, Pirro M, Ronti T, Gemelli F, Pucci G, Innocente S, Porcellati C, Mannarino E. Prognostic impact of prolonged ventricular repolarization in hypertension. Arch Intern Med 2006; 166: 909-913.

40 Fischer T, Schobel HP, Frank H, Andreae M, Schneider KT, Heusser K. Pregnancy-induced sympathetic overactivity: a precursor of preeclampsia. Eur J Clin Invest 2004; 34: 443-448.

41 Schobel HP, Fischer T, Heuszer K, Geiger H, Schmieder RE. Preeclampsia: a state of sympathetic overactivity. New Engl J Med 1996; 335: 1480-1485.

42 Levine RJ, Lam C, Qian C, Yu KF, Maynard SE, Sachs BP, Sibai BM, Epstein FH, Romero R, Thadhani R, Karumanchi SA; CPEP Study Group. Soluble endoglin and other circulating antiangiogenic factors in preeclampsia. New Eng/ J Med 2006; 355: 992-1005.

43 Levine RJ, Maynard SE, Qian C, Lim KH, England LJ, Yu KF, Schisterman EF, Thadhani R, Sachs BP, Epstein FH, Sibai BM, Sukhatme VP, Karumanchi SA. Circulating angiogenic factors and the risk of preeclampsia. New Engl J Med 2004 350: 672-683.

44 Pal GK, Shyma P, Habeebullah S, Pal P, Nanda N, Shyjus P. Vagal withdrawal and sympathetic overactivity contribute to the genesis of early-onset pregnancy-induced hypertension. Int J Hypertens 2011 2011: 361417.

45 Salles GF, Cardoso CR, Muxfeldt ES. Prognostic value of ventricular repolarization prolongation in resistant hypertension: a prospective cohort study. J Hypertens 2009; 27: 1094-1101.

46 Valensise H, Vasapollo B, Gagliardi G, Novelli GP. Early and late preeclampsia: two different maternal hemodynamic states in the latent phase of the disease. Hypertension 2008; 52: 873-880.

47 Gatzoulis MA, Till JA, Redington AN. Depolarization-repolarization inhomogeneity after repair of tetralogy of Fallot. The substrate for malignant ventricular tachycardia? Circulation 1997; 95: 401-404. 